J. Austral. Math. Soc. 22 (Series A) (1976), 343-361.

\title{
SYMMETRIC SQUARE ROOTS OF THE INFINITE IDENTITY MATRIX
}

\author{
C. E. M. PEARCE and R. B. POTTS
}

(Received 9 December 1974; revised 12 August 1975)

\begin{abstract}
Some non-trivial real, symmetric square roots of the infinite identity matrix are exhibited. These may be found either from the use of involutory integral transforms and a set of real orthonormal functions or by an algebraic factorisation procedure. The two approaches are shown to be equivalent.
\end{abstract}

Guinand (1956) has shown that square roots of the infinite identity matrix I can be generated from a Fourier kernel using biorthogonal functions. In particular, he gives an explicit expression for a family of asymmetric square roots of I, an example which generalises an earlier result of Barrucand (1950).

In this paper we use Guinand's analytical method to develop families of real, symmetric (and hence orthogonal) square roots of $I$, in the sequel referred to simply as "symmetric roots". We show that all such roots can be generated by this method, and that the method is equivalent to an algebraic factorisation procedure. Some examples and applications are also presented.

A number of texts on matrices give results on square roots of finite matrices (see, for example, Perlis (1952) Theorem 9-15), and indeed there is a literature on unilateral (polynomial) equations in a finite square matrix (MacDuffee (1946) Chapter 8 gives a bibliography). Nevertheless, apart from the work of Guinand noted above, scant attention seems to have been paid to square roots (or other fractional powers) of the infinite identity matrix. In his book on infinite matrices, Cooke (1950) mentions only some trivial examples as an exercise.

Square roots of $I$ and orthogonal matrices, or rather the associated transformations, have found application in connection with summation formulae and the corresponding inversion formulae (see Guinand (1939) and Smith (1972)).

\section{Algebraic method}

For any convenient set of non-vanishing constants $\left\{a_{n} ; n \geqq 0\right\}$ and infinite matrix $K=\left\{k_{m n} ; m, n \geqq 0\right\}$ we define formally the double generating function $F^{K}(u, v)$ by 


$$
F^{K}(u, v)=\sum_{m=0}^{\infty} \sum_{n=0}^{\infty} k_{m n} a_{m} u^{m} a_{n} v^{n}
$$

and the marginal generating function $F_{m}^{K}(z)$ by

$$
F_{m}^{K}(z)=\sum_{n=0}^{\infty} k_{m n} a_{n} z^{n}
$$

If I admits of a symmetric root $K$, then

$$
\delta_{m n}=\sum_{p=0}^{\infty} k_{p m} k_{p n}, \quad m, n \geqq 0
$$

which in terms of our generating functions reads

$$
F^{I}(u, v)=\sum_{p=0}^{\infty} F_{p}^{K}(u) F_{p}^{K}(v)
$$

Further, such a factorisation will provide a root of $I$ if the matrix $K$ generated by $\left\{F_{p}^{K}(z) ; p \geqq 0\right\}$ is symmetric. We have also

$$
F^{I}(u, v)=\sum_{p=0}^{\infty} a_{p}^{2}(u v)^{p},
$$

from which trivial solutions

$$
F^{K}(v)=(-1)^{r(p)} a_{p} v^{p}, r(p) \text { integral }
$$

are obvious, corresponding to the known roots

$$
k_{m n}=(-1)^{r(m)} \delta_{m n}, \quad m, n \geqq 0 .
$$

Less trivial solutions are also immediate. Thus, taking $a_{n}=1$, we have the decomposition

$$
\begin{aligned}
F^{I}(u, v)=(1-u v)^{-1} & =s^{2}(1-c u)^{-1}(1-c v)^{-1}\left[1-\frac{c-u}{1-c u} \frac{c-v}{1-c v}\right]^{-1} \\
& =s^{2}(1-c u)^{-1}(1-c v)^{-1} \sum_{p=0}^{\infty}\left(\frac{c-u}{1-c u}\right)^{p}\left(\frac{c-v}{1-c v}\right)^{p}
\end{aligned}
$$

where $c, s$ are subject to the constraints $c^{2}+s^{2}=1$. This relation is valid if $|u|,|v|<1$ and $|c|,|s| \leqq 1$. The decomposition suggests

$$
F_{p}^{K}(v)=s(c-v)^{p}(1-c v)^{-p-1} .
$$

Apart from a scale factor this is the generating function of certain discrete orthogonal polynomials $m_{n}\left(p ; 1, c^{2}\right)$ considered by Meixner (1941). See also Erdélyi et al. (1953) Vol. 2, pp. 225-6. The expansion can take the forms 


$$
\begin{aligned}
k_{p n} & =s \sum_{r=0}^{\min (p, n)}(-1)^{r} \frac{(p+n-r) !}{(p-r) !(n-r) ! r !} c^{p+n-2 r} \\
& =s c^{p+n} m_{n}\left(p ; 1, c^{2}\right) / n ! \\
& =s c^{p+n} \frac{(p+n) !}{p ! n !}{ }_{2} F_{1}\left(-p,-n ;-p-n ; c^{-2}\right), p, n \geqq 0,
\end{aligned}
$$

displaying the desired symmetry between $p$ and $n$. Thus (1.2) does indeed constitute a symmetric and so orthogonal square root of I. For the $k_{m n}$ of (1.2) the series $\Sigma_{p} k_{p m} k_{p n}$ does converge for all $m, n \geqq 0$, justifying the original formal relations. To keep clear the presentation of the underlying ideas we shall generally avoid in the remainder of this paper both such post hoc justifications and detailed prescriptions of domains of validity of relations stated.

Further essentially equivalent solutions arise (for a general symmetric root $K$ ) if we replace $k_{m n}$ by $(-1)^{r(m)+r(n)} k_{m n}$, which provides the same pattern of sign switches to each row and column of the matrix. $K$. In terms of the matrix $D$ defined by (1.1) $K$ has become $D^{T} K D$. Henceforth we exclude separate consideration of such possibilities.

If in place of $a_{n}=1$ we choose

$$
a_{n}=(\Gamma(\alpha+n+1))^{1 / 2}(n ! \Gamma(\alpha+1))^{-1 / 2},
$$

we obtain similarly a factorisation

$$
\begin{aligned}
(1-u v)^{-1-\alpha}= & s^{2(1+\alpha)}(1-c u)^{-1-\alpha}(1-c v)^{-1-\alpha} \\
& \times \sum_{p=0}^{\infty} \frac{\Gamma(\alpha+p+1)}{p ! \Gamma(\alpha+1)}\left(\frac{c-u}{1-c u}\right)^{p}\left(\frac{c-v}{1-c v}\right)^{p}
\end{aligned}
$$

leading to a solution

$$
\begin{aligned}
k_{p n}= & s^{1+\alpha} c^{p+n}[\Gamma(\alpha+p+1)]^{1 / 2}[p ! n ! \Gamma(\alpha+n+1)]^{-1 / 2} m_{n}\left(p ; 1+\alpha, c^{2}\right) \\
= & s^{1+\alpha} c^{p+n} \Gamma(p+n+\alpha+1)(p ! n ! \Gamma(\alpha+p+1) \Gamma(\alpha+n+1)]^{-1 / 2} \\
& \times{ }_{2} F_{1}\left(-p,-n ;-p-n-\alpha ; c^{-2}\right) .
\end{aligned}
$$

We deduce also that

$$
F^{K}(u, v)=s^{1+\alpha}[1-c(u+v)+u v]^{-1-\alpha}
$$

In view of the discussion of the following sections it is perhaps worth noting that the factorisation method, while set up here to deal with symmetric roots, can also be adapted to the context of asymmetric roots. 


\section{Analytical method}

Suppose that $K(x, y)=K(y, x)$ is the symmetric kernel of an involutory transformation, exhibited by

$$
f(x)=\int K(x, y) g(y) d y, \quad g(y)=\int K(x, y) f(x) d x,
$$

and further that

$$
f(x)=\sum_{0}^{\infty} a_{m} \varphi_{m}(x), \quad g(y)=\sum_{0}^{\infty} b_{n} \varphi_{n}(y)
$$

are expansions of $f$ and $g$ in terms of a set of real orthonormal functions $\left\{\varphi_{m}\right\}$. Then, by a formal argument,

$$
\begin{aligned}
a_{m} & =\int f(x) \varphi_{m}(x) d x \\
& =\iint \varphi_{m}(x) K(x, y) g(y) d x d y \\
& =\sum_{n} \iint \varphi_{m}(x) K(x, y) \varphi_{n}(y) d x d y b_{n},
\end{aligned}
$$

that is,

$$
a_{m}=\sum_{n} k_{m n} b_{n}
$$

where

$$
k_{m n}=\iint \varphi_{m}(x) K(x, y) \varphi_{n}(y) d x d y .
$$

In the same way the relation

$$
b_{n}=\sum_{m} k_{n m} a_{m}
$$

follows so that the matrix $K=\left(k_{m n}\right)$ satisfies the relations

$$
K^{2}=I, \quad K^{T}=K
$$

This formal argument follows that of Guinand except that he considers the special case when $K(x, y)=K(x y)$ is a Fourier kernel and employs biorthogonal functions $\left\{\varphi_{m}(x)\right\},\left\{\psi_{m}(x)\right\}$. The use of biorthogonal functions destroys the symmetry property of the matrix $K$.

In particular, take as kernel the Bessel function $J_{\alpha}\left[2(x y)^{\frac{1}{2}}\right]$ so that the involutory transformation is the Hankel transform. As a set of orthonormal functions over $(0, \infty)$ choose

$$
\varphi_{m}^{\alpha}(t ; x)=\left[\frac{m !}{\Gamma(\alpha+m+1)}\right]^{\frac{1}{2}} t^{\frac{1}{2}(1+\alpha)} x^{\frac{1}{2} \alpha} \exp \left(-\frac{1}{2} t x\right) L_{m}^{\alpha}(t x), \quad m \geqq 0,
$$


where the $L_{m}^{\alpha}$ are the generalised Laguerre polynomials. Then by $(2.1)$ the elements $k_{m n}^{\alpha}(t)$ of the matrix $K^{\alpha}(t)$ can be expressed as double integrals by

$$
\begin{aligned}
k_{m n}^{\alpha}(t)= & {\left[\frac{m ! n !}{\Gamma(\alpha+m+1) \Gamma(\alpha+n+1)}\right] t^{1+\alpha} . } \\
& \int_{0}^{\infty} \int_{0}^{\infty} x^{\frac{1}{2} \alpha} \exp \left(-\frac{1}{2} t x\right) L_{m}^{\alpha}(t x) J_{\alpha}\left[2(x y)^{\frac{1}{2}}\right] y^{\frac{1}{2} \alpha} \exp \left(-\frac{1}{2} t y\right) L_{n}^{\alpha}(t y) d x d y .
\end{aligned}
$$

Following a transformation $(t x)^{1 / 2}=u, 2(y / t)^{1 / 2}=v$, the integration on $x$ can be evaluated through the Hankel transform

$$
\int_{0}^{\infty} u^{\alpha+\frac{1}{2}} e^{-\frac{1}{2} u^{2}} L_{n}^{\alpha}\left(u^{2}\right) J_{\alpha}(u v)(u v)^{\frac{1}{2}} d u=(-1)^{n} e^{-\frac{1}{2} v^{2}} v^{\alpha+\frac{1}{2}} L_{n}^{\alpha}\left(v^{2}\right)
$$

(Erdélyi et al. (1954), Vol. 2, 8.9(3)) to yield

$$
\begin{aligned}
k_{m n}^{\alpha}(t)= & {\left[\frac{m ! n !}{\Gamma(\alpha+m+1) \Gamma(\alpha+n+1)}\right]^{\frac{1}{2}} 2^{1+\alpha}(-1)^{n} . } \\
& \int_{0}^{\infty} y^{\alpha} \exp \left(-y\left(\frac{t}{2}+\frac{2}{t}\right)\right) L_{n}^{\alpha}(4 y / t) L_{m}^{\alpha}(t y) d y .
\end{aligned}
$$

The second integration may be found by use of the Laplace transform

$$
\begin{aligned}
\int_{0}^{\infty} e^{-s y} y^{\alpha} L_{n}^{\alpha}(\lambda y) L_{m}^{\alpha}(\kappa y) d y \\
=\frac{\Gamma(m+n+\alpha+1)}{m ! n !} \frac{(s-\lambda)^{n}(s-\kappa)^{m}}{s^{m+n+\alpha+1}} \\
\quad{ }_{2} F_{1}\left(-m,-n ;-m-n-\alpha ; \frac{s(s-\lambda-\kappa)}{(s-\lambda)(s-\kappa)}\right)
\end{aligned}
$$

(Erdélyi et al. (1954), Vol. 2, 4.11 (35)), as

$$
\begin{aligned}
k_{m n}^{\alpha}(t)= & {\left[\frac{p^{1+\alpha} q^{m+n}}{m ! n ! \Gamma(\alpha+m+1) \Gamma(\alpha+n+1)}\right]^{\frac{1}{2}} \Gamma(m+n+\alpha+1) } \\
& \times{ }_{2} F_{1}\left(-m,-n ;-m-n-\alpha ; q^{-1}\right)
\end{aligned}
$$

with

$$
p=16 t^{2}\left(4+t^{2}\right)^{-2}>0, \quad q=1-p=\left(4-t^{2}\right)^{2}\left(4+t^{2}\right)^{-2}>0 .
$$

The symmetry of $K^{\alpha}(t)$ is evident from equation (2.5) and its orthogonality can be verified by appeal to the identity

(2.7) $\Gamma(l+n+\alpha+1){ }_{2} F_{1}\left(-l,-n ;-l-n-\alpha ; q^{-1}\right)=\Gamma(\alpha+l+1) m_{n}(l ; 1+a, q)$ in the Meixner polynomials. Equation (2.4) can be regarded as an integral expression for these polynomials. 
Equation (2.5) is, of course, simply the solution (1.4) with $c=q^{\frac{1}{2}}, s=p^{\frac{1}{1}}$. When $\alpha=0$, the matrix entries simplify for suitable choices of $t$. Thus for $t=1$, so that $p=16 / 25$ and $q=9 / 25$, the matrix takes the form

$$
K^{0}(1)=\frac{4}{5} \quad\left[\begin{array}{cccc}
1 & \frac{3}{5} & \left(\frac{3}{5}\right)^{2} & \ldots \\
\frac{3}{5} & 2\left(\frac{3}{5}\right)^{2}-1 & 3\left(\frac{3}{5}\right)^{3}-2\left(\frac{3}{5}\right) & \ldots \\
\left(\frac{3}{5}\right)^{2} & 3\left(\frac{3}{5}\right)^{3}-2\left(\frac{3}{5}\right) & 6\left(\frac{3}{5}\right)^{4}-6\left(\frac{3}{5}\right)^{2}+1 & \ldots \\
. & . & . & \ldots
\end{array}\right]
$$

\section{Correspondence between the methods}

We now correlate our two approaches through the following constructive principle:

Every symmetric root $K$ corresponds formally to a Guinand scheme.

Proof. For any complete orthonormal family $\left\{\phi_{m} ; m \geqq 0\right\}$ on $(0, \infty)$, consider the function

$$
K(x, y)=\sum_{m} \sum_{n} k_{m n} \phi_{m}(x) \phi_{n}(y)
$$

The symmetry of $\left(k_{m n}\right)$ entails $K(x, y)=K(y, x)$, that is, $K(x, y)$ is symmetric. From the orthonormality of the $\phi_{m}$, we have immediately that $K(x, y)$ satisfies

$$
k_{m n}=\int_{0}^{\infty} \int_{0}^{\infty} \phi_{m}(x) \phi_{n}(y) K(x, y) d x d y .
$$

Further, define

$$
\Phi_{m}(y)=\sum_{n} k_{m n} \phi_{n}(y)
$$

Then

$$
k_{m n}=\int_{0}^{\infty} \Phi_{m}(y) \phi_{n}(y) d y
$$

and so

$$
\int_{0}^{\infty} \phi_{m}(x)\left[\Phi_{n}(x)-\int_{0}^{\infty} \phi_{n}(y) K(x, y) d y\right] d x=0
$$

for all $m$, from which we see that 


$$
\Phi_{n}(x)=\int_{0}^{\infty} \phi_{n}(y) K(x, y) d y .
$$

We can readily deduce from $K^{2}=I$ that also

$$
\phi_{n}(x)=\int_{0}^{\infty} \Phi_{n}(y) K(x, y) d y .
$$

As an arbitrary analytic function can be expressed as a linear combination of the $\phi_{n}$ (or the $\Phi_{n}$ ), it follows from (3.1) and (3.2) that $K$ is a Fourier kernel, completing the proof.

Consideration of the equation

$$
\iint \phi_{m}(x)[K(x, y)-H(x, y)] \phi_{n}(y) d x d y=0
$$

gives easily that for a specific choice of orthonormal family the kernel in the integral representation is unique. By virtue of the relations

$$
\sum_{p} k_{m p} k_{n p}=\delta_{m n}
$$

the family $\left\{k_{m x} ; m=0,1, \cdots\right\}$ for $x$ a non-negative integer is orthonormal and the decomposition

$$
k_{m n}=\sum_{x=0}^{\infty} \sum_{y=0}^{\infty} k_{x y} k_{m x} k_{n y}
$$

is always trivially available as a degenerate integral representation. The construction makes it clear that the selection of kernel and orthonormal family in the integral representation will not be unique. Fresh representations are often available by simple change of variable - the introduction of $t$ in equation (2.4) can in particular be regarded as a change of variable. In the case $\alpha=-\frac{1}{2}$ in the example above, let us make the transformations

$$
x=\frac{1}{2} w^{2}, \quad y=\frac{1}{2} z^{2} .
$$

From the relations

$$
\begin{aligned}
J_{-\frac{1}{2}}(u) & =\left(\frac{2}{\pi u}\right)^{\frac{1}{2}} \cos u \\
L_{m}^{-1 / 2}\left(\frac{1}{2} u\right) & =(m !)^{-1}\left(-\frac{1}{2}\right)^{m} \operatorname{He}_{2 m}(u) \\
\Gamma(v) \Gamma\left(v+\frac{1}{2}\right) & =\pi^{\frac{1}{2}} 2^{1-2 v} \Gamma(2 v)
\end{aligned}
$$

it quickly follows that

$$
\begin{aligned}
k_{m n}^{-1 / 2}(t)= & \frac{(-1)^{m+n}}{[(2 m) !(2 n) !]^{2}} \frac{2 t^{\frac{1}{2}}}{\pi} \\
& \exp \left(-\frac{1}{4} t w^{2}\right) H e_{2 m}\left(t^{\frac{1}{2}} w\right) \cos w z \exp \left(-\frac{1}{4} t z^{2}\right) H e_{2 n}\left(t^{\frac{1}{2}} z\right) d w d z .
\end{aligned}
$$


Thus $k_{m n}^{-1 / 2}(t)$ now utilises the Fourier cosine kernel $(2 / \pi)^{\frac{1}{2}} \cos w$ and takes for orthonormal functions

$$
\varphi_{m}(t ; w)=(-1)^{m}[(2 m) !]^{-\frac{1}{2}}(2 t / \pi)^{\frac{1}{4}} \exp \left(-\frac{1}{4} t w^{2}\right) H e_{2 m}\left(t^{\frac{1}{2}} w\right) .
$$

A similar result arises for $\alpha=\frac{1}{2}$ using the Fourier sine kernel and the odd order Hermite polynomials.

Despite the completeness of the Guinand scheme, relatively few Fourier kernels together with their associated orthonormal functions are known, and many of the integrals of orthonormal functions are difficult to evaluate. Sometines the derivation of square roots $K$ through the factorisation procedure involves quite simple algebra. Thus choosing $a_{n}=(n !)^{-\frac{1}{2}}$, we find

$$
\begin{aligned}
F^{I}(u, v) & =\exp (u v) \\
& =\exp \left[(c-u)(c-v)+c(u+v)-c^{2}\right] \\
& =\sum_{p=0}^{\infty} \exp \left(-\frac{1}{2} c^{2}\right) \exp (c u) \frac{(c-u)^{p}}{(p !)^{\frac{1}{2}}} \exp \left(-\frac{1}{2} c^{2}\right) \exp (c v) \frac{(c-v)^{p}}{(p !)^{\frac{1}{2}}}
\end{aligned}
$$

for $c$ arbitrary, suggesting

$$
F_{m}^{K}(v)=\exp \left(-\frac{1}{2} c^{2}\right) \exp (c v) \frac{(c-v)^{m}}{(m !)^{2}} .
$$

$F_{m}^{K}(v)$ can be recognised as a multiple of a generating function of the Charlier polynomials (see Erdélyi et al. (1953), Vol. 2, p. 226) and leads to

$$
\begin{aligned}
k_{m n} & =\exp \left(-\frac{1}{2} c^{2}\right) c^{m+n}(m ! n !)^{-\frac{1}{2}} c_{n}\left(m ; c^{2}\right) \\
& =\exp \left(-\frac{1}{2} c^{2}\right) c^{m+n}(m ! n !)^{-\frac{1}{2}}{ }_{2} F_{0}\left(-m,-n ;-;-c^{-2}\right),
\end{aligned}
$$

displaying an appropriate symmetry in $m$ and $n$.

Equation (3.4) can also be obtained by a limiting process from (1.4) and the identity

$$
\lim _{b \rightarrow x} b^{-n} m_{n}(x ; b, a / b)=c_{n}(x ; a)
$$

(reference as above). However, there is no obvious corresponding non-trivial integral representation, the constructive theorem (with natural choice of orthonormal family) and the limiting form of equation (2.4) both giving the degenerate representation, which is simply the standard orthogonality property of the Charlier polynomials.

The practical inequivalence of the algebraic and analytical approaches can be utilised as a source of unexpected but premeditated identities. For instance, take $\left(k_{m n}\right)$ to be as in the basic Guinand scheme example of section two with 
kernel $J_{\alpha}\left(2(x y)^{\frac{1}{2}}\right)$ and orthonormal functions given by (2.3), but let us deviate from the choice (1.3) of $a_{n}$ selected in the algebraic approach and instead set

$$
a_{n}=[n ! \Gamma(\alpha+n+1)]^{-\frac{1}{2}} .
$$

Then

$$
F^{I}(u, v)=\sum_{m=0}^{\infty} \frac{(u v)^{m}}{m ! \Gamma(\alpha+m+\mathrm{r})}=(u v)^{-\frac{1}{2} \alpha} I_{\alpha}\left(2(u v)^{\frac{1}{2}}\right),
$$

where $I_{\alpha}$ represents a modified Bessel function of the first kind. Also, on forming double generating functions and evaluating the integrals, we find that

$$
\begin{aligned}
F^{K}(u, v)= & t \int_{0}^{\infty} \int_{0}^{\infty}(u v)^{-\frac{1}{2} \alpha} \exp (u+v) J_{\alpha}\left(2(t x u)^{\frac{1}{1}}\right) \exp \left(-\frac{1}{2} t x\right) J_{\alpha}\left(2(x y)^{\frac{1}{1}}\right) \\
& \times J_{\alpha}\left(2(t y v)^{\frac{1}{2}}\right) \exp \left(-\frac{1}{2} t y\right) d x d y \\
= & p^{\frac{1}{2(1+\alpha)}} \exp \left[(u+v) q^{\frac{1}{2}}\right](\Gamma(1+\alpha))^{-1}{ }_{0} F_{1}(-; 1+\alpha ;-u v p),
\end{aligned}
$$

where $p, q$ are given by (2.6). This leads to

$$
\begin{aligned}
F_{m}^{K}(u) & =p^{\frac{1}{2}(1+\alpha)} \exp \left(u q^{\frac{1}{2}}\right) q^{\frac{1}{2} m}[\Gamma(\alpha+m+1) / m !]^{\frac{1}{1}} F_{1}\left(-m ; 1+\alpha ; u p q^{-\frac{1}{2}}\right) \\
& =p^{\frac{1}{2}(1+\alpha)} \exp \left(u q^{\frac{1}{2}}\right) q^{\frac{1}{2} m}[m ! / \Gamma(\alpha+m+1)]^{\frac{1}{2}} L_{m}\left(u p q^{-\frac{1}{2}}\right) .
\end{aligned}
$$

Thus we have the identity

$$
\begin{aligned}
& (u v)^{-\frac{1}{2} \alpha} I_{\alpha}\left(2(u v)^{\frac{1}{2}}\right)=\sum_{m=0}^{\infty} F_{m}^{K}(u) F_{m}^{K}(v) \\
& =\sum_{m=0}^{\infty} p^{1+\alpha} \exp \left[(u+v) q^{\frac{1}{2}}\right] q^{m} m ![\Gamma(1+\alpha+m)]^{-1} L_{m}\left(u p q^{-\frac{1}{2}}\right) L_{m}\left(v p q^{-\frac{1}{2}}\right) .
\end{aligned}
$$

This striking decomposition manifesting symmetry between the two argument components of $I$ is just the Hille-Hardy formula

$$
\begin{aligned}
\sum_{n=0}^{\infty} n !(\Gamma(\alpha+n+1))^{-1} L_{n}^{\alpha}(x) L_{n}^{\alpha}(y) z^{n} \\
\quad=(1-z)^{-1} \exp [-z(x+y) /(1-z)](x y z)^{-\frac{1}{2} \alpha} I_{\alpha}\left[2(x y z)^{\frac{1}{2}} /(1-z)\right],
\end{aligned}
$$

as can be seen from the substitutions $u=x z^{\frac{1}{2}} /(1-z), v=y z^{\frac{1}{2}} /(1-z)$, with $z=q$. For discussion of the Hille-Hardy formula see Erdélyi et al. (1953), Vol. 3, 19.12(15), Hardy (1932) and Watson (1933b). The same method can be used to rovide other symmetric decompositions of $I_{\alpha}$; thus, for the same orthonormal amily and a kernel $J_{\nu}, \nu \neq \alpha$, we derive a straightforward, though somewhat zumbersome, extension of the Hille-Hardy expansion of the modified Bessel unction in terms of Laguerre functions. 


\section{Generalised Hermite polynomials}

In the previous section we noted for $\alpha= \pm \frac{1}{2}$ an alternative representation to (2.4) in terms of Fourier sine (cosine) transforms. The representation employing Hermite polynomials can be extended to hold for any $\alpha$ (with kernel $w^{\frac{1}{2}} J_{\alpha}(w)$ ) if the Hermite polynomials are suitably generalised. Although the naturalness of a generalisation is suggested by a number of results in the theory of special functions, the authors have been unable to locate any version of one in the literature. The following generalisation seems useful; for convenience, we refer to the functions $H_{n}^{\alpha}$ rather than functions $\mathrm{He}_{n}^{\alpha}$ and define for real $x$

$$
\begin{aligned}
H_{2 n}^{\alpha}(x) & =2^{2 n} \Psi\left(-n, \alpha+1 ; x^{2}\right) \\
H_{2 n+1}^{\alpha}(x) & =2^{2 n+1} x \Psi\left(-n, \alpha+2 ; x^{2}\right)
\end{aligned}
$$

where $\Psi$ represents the confluent hypergeometric function. With these definitions

$$
H_{n}^{-1 / 2}(x)=H_{n}(x),
$$

and $H_{n}^{\alpha}$ is of proper degree and parity as indicated by its subscript. The polynomials are orthonormal on $(-\infty, \infty)$ for the weight function

$$
|x|^{2 \alpha+1} e^{-x^{2}}
$$

with orthonormalised forms

$$
\begin{aligned}
& 2^{-\frac{1}{2}} h_{n}^{\alpha}(x)=2^{-\frac{1}{2}} \exp \left(-\frac{1}{2} x^{2}\right)|x|^{\alpha+\frac{1}{2}} 2^{-2 n}[n ! \Gamma(\alpha+n+1)]^{-\frac{1}{2}} H_{2 n+1}^{\alpha}(x) \\
& 2^{-\frac{1}{2}} g_{n}^{\alpha}(x)=2^{-\frac{1}{2}} \exp \left(-\frac{1}{2} x^{2}\right)|x|^{\alpha+\frac{1}{2}} 2^{-(2 n+1)}[n ! \Gamma(\alpha+n+2)]^{-\frac{1}{2}} H_{2 n+1}^{\alpha}(x)
\end{aligned}
$$

respectively for $H_{2 n}^{\alpha}(x)$ and $H_{2 n+1}^{\alpha}(x)$. In terms of the $H_{n}^{\alpha}$, the entities $k_{m n}$ in the example can for general $\alpha$ now be expressed in Guinand form through the functions $h_{n}^{\alpha}$ (or $g_{n}^{\alpha-1}$ ) orthonormal on $(0, \infty)$.

The definitions lead to alternative expressions

$$
\begin{gathered}
H_{2 n}^{\alpha}(x)=(-1)^{n} 2^{2 n} n ! L_{n}^{\alpha}\left(x^{2}\right) \\
H_{2 n+1}^{\alpha}(x)=(-1)^{n} 2^{2 n+1} n ! x L_{n}^{1+\alpha}\left(x^{2}\right),
\end{gathered}
$$

from which salient properties of the $H_{n}^{\alpha}$ can be derived via the properties of the Laguerre polynomials. Thus the identities

$$
\begin{gathered}
L_{n}^{\alpha}(y)=L_{n}^{1+\alpha}(y)-L_{n+1}^{1+\alpha}(y) \\
y L_{n}^{1+\alpha}(y)=(n+\alpha+1) L_{n}^{\alpha}(y)-(n+1) L_{n+1}^{\alpha}(y)
\end{gathered}
$$

generate the recurrence relations

$$
H_{2 n+1}^{\alpha}(x)-2 x H_{2 n}^{\alpha}+4 n H_{2 n-1}^{\alpha}(x)=0
$$


and

$$
H_{2 n+2}^{\alpha}(x)-2 x H_{2 n+1}^{\alpha}(x)+4(n+\alpha+1) H_{2 n}^{\alpha}(x)=0,
$$

whilst the differential equation for $L_{n}^{\alpha}(y)$,

$$
y L^{\prime \prime}+(\alpha+1-y) L^{\prime}+n L=0
$$

provides

$$
x\left(H_{2 n}^{\alpha}\right)^{\prime \prime}(x)+\left(2 \alpha+1-2 x^{2}\right)\left(H_{2 n}^{\alpha}\right)^{\prime}(x)+4 x n H_{2 n}^{\alpha}(x)=0
$$

and

$$
\begin{aligned}
x\left(H_{2 n+1}^{\alpha}\right)^{\prime \prime}(x)+(2 \alpha+1 & \left.-2 x^{2}\right)\left(H_{2 n+1}^{\alpha}\right)^{\prime}(x) \\
+ & {[(2 n+1) 2 x-(2 \alpha+1) / x] H_{2 n+1}^{\alpha}(x)=0 . }
\end{aligned}
$$

The divergence in form between (4.3), (4.4) and (4.3'), (4.4') for even and odd subscripted $H_{n}$ contrasts sharply with the behaviour of classical orthogonal polynomials. Bochner (1929) has shown that the classical polynomials $f_{n}(x)$ are completely characterised by satisfying differential equations of the form

$$
A(x) y^{\prime \prime}+B(x) y^{\prime}+\lambda_{n} y=0,
$$

where $A$ and $B$ are independent of $n$ and $\lambda_{n}$ is independent of $x$. As the generalised Hermite polynomials do not satisfy such relation, we see that despite relations (4.1) and (4.2) we should not take them as being in any real sense equivalent to a classical system. We note also that this entails the absence of a Rodrigues' formula for the generalised Hermite polynomials, as Tricomi (1948) has shown that possessing such a formula again completely characterises the classical orthogonal polynomials.

\section{More general solutions}

Further symmetric roots $K$ can be constructed by the use of different Fourier kernels. The fullest reference to collections of Fourier transforms is perhaps that given in Chapter 8 of Titchmarsh (1937), which notes in particular the very general work of Fox (1929) and Kuttner (1934). Some subtle but simple transforms have also been found by Guinand (1942), (1950).

Consider the Meijer $G$-function specified by

$$
G_{2 n 2 m}^{m n}(x)=G_{2 n 2 m}^{m n}\left(x \mid \begin{array}{l}
a_{1}, \cdots, a_{n},-a_{n}, \cdots,-a_{1} \\
b_{1}, \cdots, b_{m},-b_{m}, \cdots,-b_{1}
\end{array}\right)
$$

We have formally that the Mellin transform of a general Meijer $G$-function $G_{p q}^{m n}\left(x \mid \begin{array}{l}a_{1}, \cdots, a_{p} \\ b_{1}, \cdots, b_{q}\end{array}\right)$ is 


$$
\frac{\prod_{i=1}^{m} \Gamma\left(b_{i}+s\right) \prod_{i=1}^{n} \Gamma\left(1-a_{j}-s\right)}{\prod_{i=m+1}^{q} \Gamma\left(1-b_{j}-s\right) \prod_{j=n+1}^{p} \Gamma\left(a_{j}+s\right)}
$$

(for a discussion see Erdélyi et al. (1953)). Hence the Mellin transform $M(s)$ of the function given in (5.1) satisfies $M(s) M(1-s)=1$. By a result due to Watson (1933a) and Hardy and Titchmarsh (1933), $G$ is thus a Fourier kernel. This rôle for the $G$-function does not appear to have been previously noted in the literature, although the results are implicit in the work of Kuttner (1934).

With a double application of the relation

$$
G_{h k}^{m n}\left(\begin{array}{l|l}
w t & \begin{array}{l}
a_{1}, \cdots, a_{h} \\
b_{1}, \cdots, b_{k}
\end{array}
\end{array}\right) t^{-\beta} e^{-s w t} d t=(w s)^{\beta-1} G_{h+1 k}^{m+1}\left(\begin{array}{l|c}
s^{-1} & \begin{array}{c}
\beta, a_{1}, \cdots, a_{h} \\
b_{1}, \cdots, b_{k}
\end{array}
\end{array}\right)
$$

and $a_{p}$ set equal to $\left(\Gamma(\alpha+p+1) \frac{1}{k^{2}}(\Gamma(1+\alpha) p !)^{-\frac{1}{2}}\right.$, the double generating function for the symmetric root $K$ deriving from the orthonormal functions $\varphi_{p}^{\alpha}(t ; x)$ given by (2.3) with this kernel is seen to be

$$
\begin{gathered}
F^{K}(u, v)=(\Gamma(1+\alpha))^{-1}[(1-u)(1-v)]^{-1-\alpha} \int_{0}^{\infty} \int_{0}^{\infty} \exp \left[-\frac{1}{2} x(1+u) /(1-u)\right] \\
t^{1+\alpha} x^{\frac{1}{2} \alpha} G_{2 n 2 m}^{m n}(x y) \exp \left[-\frac{1}{2} y(1+v) /(1-v)\right] y^{\frac{1}{2} \alpha} d x d y
\end{gathered}
$$

or

$$
\begin{aligned}
F^{K}(u, v)= & 2^{2+\alpha}(\Gamma(1+\alpha))^{-1}[t(1+u)(1+v)]^{-1}\left[\left(1-u^{2}\right)\left(1-v^{2}\right)\right]^{-\frac{1}{2} \alpha} \\
& G_{2 n+2}^{m} \frac{n+2}{2 m}\left(\frac{4(1-u)(1-v)}{t(1+u)(1+v)} \mid \begin{array}{c}
-\frac{1}{2} \alpha,-\frac{1}{2} \alpha, a_{1}, \cdots,-a_{1} \\
b_{1}, \cdots,-b_{1}
\end{array}\right)
\end{aligned}
$$

In the general case, the matrices generated by this procedure are of a very complicated nature indeed. When $n=0, m=1$ and $b_{1}=\frac{1}{2} \alpha$, the kernel reduces to

$$
G_{02}^{10}\left(x \mid \overline{\frac{1}{2} \alpha,-\frac{1}{2} \alpha}\right)=J_{\alpha}\left(2 x^{\frac{1}{2}}\right)
$$

and the $G$-function in (5.2) is

$$
\begin{aligned}
& G_{22}^{12}\left(\frac{4(1-u)(1-v)}{t^{2}(1+u)(1+v)} \mid \begin{array}{c}
-\frac{1}{2} \alpha,-\frac{1}{2} \alpha \\
\frac{1}{2},-\frac{1}{2} \alpha
\end{array}\right) \\
&=G_{11}^{11}\left(\frac{4(1-u)(1-v)}{t^{2}(1+u)(1+v)} \mid \begin{array}{c}
-\frac{1}{2} \alpha \\
\frac{1}{2} \alpha
\end{array}\right) \\
&= \Gamma(1+\alpha)\left[4 t^{-2}(1-u)(1-v)(1+u)^{-1}(1+v)^{-1}\right]^{\frac{1}{2} \alpha} \\
&{ }_{1} F_{0}\left(1+\alpha ;-;-4 t^{-2}(1-u)(1-v)(1+u)^{-1}(1+v)^{-1}\right)
\end{aligned}
$$


so that

$$
F^{K}(u, v)=\left(4 t /\left(4+t^{2}\right)\right)^{1+\alpha}\left[1-\left(4-t^{2}\right)\left(4+t^{2}\right)^{-1}(u+v)+u v\right]^{-1-\alpha},
$$

the solution already obtained as (1.5).

\section{Eigenvalue problem}

The possible eigenvalues of $K$ satisfying (2.2) are 1 or -1 with correspondng eigenvectors related to self-reciprocal and skew-reciprocal functions ;atisfying

$$
f(x)= \pm \int K(x, y) f(y) d y .
$$

For the matrix $K^{\alpha}(t)$ arising from the Hankel transform and exhibited in $2.5)$, the solution of the eigenvalue problem proceeds as follows. It is evident rom (2.5) that in the limiting case $q \rightarrow 0$ (or $t \rightarrow 2$ ), $k_{m n}^{\alpha}(t) \rightarrow(-1)^{n} \delta_{m n}$ giving a liagonal matrix $D$ with elements 1 and -1 alternating on the diagonal. The imiting expression for the orthonormal functions given by (2.3) is

$$
\varphi_{m}^{\alpha}(2 ; x)=\left[\frac{m !}{\Gamma(m+1+\alpha)}\right]^{\frac{1}{2}} 2^{\frac{1}{2}(1+\alpha)} x^{\frac{1}{2} \alpha} e^{-x} L_{m}^{\alpha}(2 x)
$$

which for $m$ even are self-reciprocal and $m$ odd are skew-reciprocal functions of he Hankel transform. In fact, neglect of an inconsequential multiplicative factor ;ives the identity

$$
x^{\frac{1}{2} \alpha} e^{-x} L_{m}^{a}(2 x)=(-1)^{m} \int_{0}^{\infty} J_{\alpha}\left[2(x y)^{\frac{1}{2}}\right] y^{\frac{1}{2} \alpha} e^{-y} L_{m}^{\alpha}(2 y) d y .
$$

To derive the eigenvectors of $K^{\alpha}(t)$ we only need to specify the expansion

$$
\varphi_{m}^{\alpha}(t, x)=\sum_{n} l_{m n}^{\alpha} \varphi_{n}^{\alpha}(2 ; x)
$$

The coefficients $l_{m n}^{\alpha}$ are the elements of an orthogonal matrix $L^{\alpha}(t)$ which liagonalises $K^{\alpha}(t)$, and the columns of $L^{\alpha}(t)$ are the required eigenvectors.

Explicit calculation gives the non-symmetric elements

$$
\begin{gathered}
l_{m n}^{\alpha}(t)=(-1)^{n}\left[\frac{r^{1+\alpha} s^{m+n}}{m ! n ! \Gamma(m+1+\alpha) \Gamma(n+1+\alpha)}\right]^{\frac{1}{2}} \Gamma(m+n+1+\alpha) . \\
{ }_{2} F_{1}\left(-m,-n ;-m-n-\alpha ; s^{-1}\right)
\end{gathered}
$$

vith

$$
\begin{gathered}
r=8 t(2+t)^{-2}>0 \\
s=1-r=(2-t)^{-2}(2+t)^{-2}>0 .
\end{gathered}
$$


Comparison with (2.5) shows that the matrix $L^{\alpha}(t)$ is simply

$$
L^{\alpha}(t)=K^{\alpha}\left((2 t)^{\frac{1}{2}}\right) D
$$

since replacing $t$ by $(2 t)^{\frac{1}{2}}$ transforms $q$ given by (2.6) to $s$ given by (6.1). The diagonalising relation

$$
L^{\alpha}(t)^{T} K^{\alpha}(t) L^{\alpha}(t)=D
$$

together with (6.2) leads to

$$
K^{\alpha}\left((2 t)^{\frac{1}{2}}\right) K^{\alpha}(t) K^{\alpha}\left((2 t)^{\frac{1}{2}}\right)=D,
$$

that is, $K^{\alpha}\left((2 t)^{\frac{1}{2}}\right)$ is also a diagonalising matrix for $K^{\alpha}(t)$. This identity,

$$
K^{\alpha}(t) K^{\alpha}\left((2 t)^{\frac{1}{2}}\right)=K^{\alpha}\left((2 t)^{\frac{1}{2}}\right) D,
$$

written in explicit component form, is a bilinear relation for hypergeometric functions:

$$
\begin{aligned}
\sum_{p=0}^{\infty} & \left(\frac{2 s}{1+s}\right)^{p} \frac{\Gamma(p+m+1+\alpha) \Gamma(p+n+1+\alpha)}{p ! \Gamma(p+1+\alpha)} . \\
& { }_{2} F_{1}\left(-p,-m ;-p-m-\alpha ;(1+s)^{2} /(4 s)\right) \cdot{ }_{2} F_{1}\left(-p,-n ;-p-n-\alpha ; s^{-1}\right) \\
= & (-1)^{n}(1+s)^{m+1+\alpha} 2^{-m}(1-s)^{-1-\alpha} \Gamma(m+n+1+\alpha) . \\
& { }_{2} F_{1}\left(-m,-n ;-m-n-\alpha ; s^{-1}\right),
\end{aligned}
$$

a particular example of the general bilinear relations derived by Meixner (1941).

We note for reference that the matrix $L$ associated with the numerica example ending section two has the form:

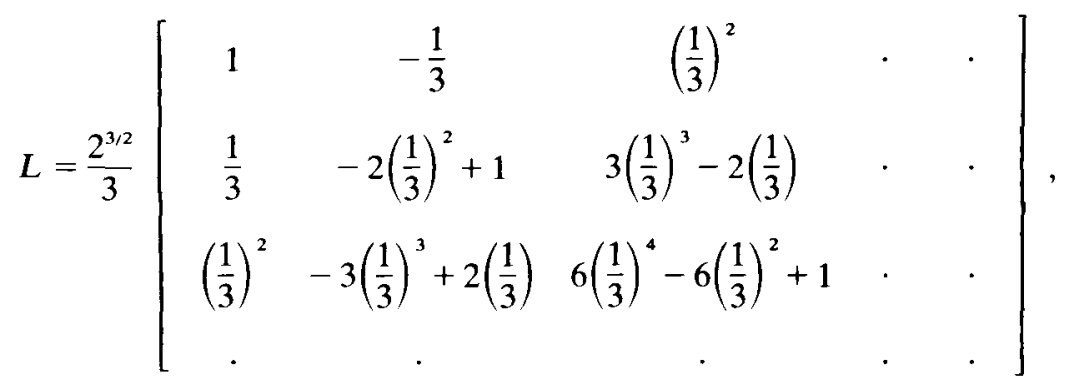

with

$$
\left.l_{m n}^{0}(1)=-1\right)^{n} 2^{3 / 2}(1 / 3)^{m+n+1} \frac{(m+n) !}{m ! n !}{ }_{2} F_{1}(-m,-n ;-m-n ; 9) .
$$

In the case previously instanced of a Fourier cosine transform, a simila analysis pivots on the functions $\varphi_{m}(t ; w)$ given by (3.3) with $t$ set equal to 2 
These functions are easily verified to be the parabolic cylinder functions, well-known examples of self- and skew-reciprocal functions of the Fourier cosine transform.

\section{Discussion}

The remarkable result (6.3), that the diagonalisation of $K^{\alpha}(t)$ is effected by the orthogonal matrix $K^{\alpha}\left((2 t)^{\frac{1}{2}}\right)$, also follows from more general considerations:

We have trivially that if $K$ is a square root of $I$, then so is an arbitrary similarity transform $S^{-1} K S$. When both $K$ and $S$ are orthogonal, $S^{-1} K S$ will be symmetric. Thus further symmetric roots can be constructed from a given one via the orthogonality transform.

Suppose

$$
k_{m n}=\iint K(x, y) \phi_{m}(x) \phi_{n}(y) d x d y
$$

exhibits an integral representation of a root in terms of an orthonormal family $\left.\phi_{m}(x)\right\}$ and that $\left\{\psi_{m}(x)\right\}$ is a complete orthonormal set of self- and skeweciprocal functions for the kernel $K(x, y)$, for definiteness, say

$$
\psi_{m}(x)=(-1)^{\prime(m)} \int K(x, y) \psi_{m}(y) d y, \quad r(m) \quad \text { integral }
$$

If

$$
\phi_{m}(x)=\sum_{n} l_{m n} \psi_{n}(x),
$$

ubstitution from the last two relations in equation (7.1) gives

$$
K=L D L^{T},
$$

vhere $L=\left(l_{m n}\right)$ and $D=\operatorname{diag}\left((-1)^{r(m)}\right)$.

Writing $\phi=\left(\phi_{m}(x)\right), \psi=\left(\psi_{m}(x)\right)$, we have also

$$
\phi=L \psi
$$

o that the relations

$$
\int \phi \phi^{T} d x=I=\int \psi \psi^{T} d x
$$

Irovide

$$
L L^{T}=I \text {. }
$$

Finally, set $X=L D$. Then from (7.2), (7.3) we have immediately

$$
K=X D X^{T} \quad \text { and } \quad X X^{T}=I \text {. }
$$


The matrix $K$ thus derives from the "elementary" root $D$ of $I$ through an orthogonality transform $X . X$ must clearly contain all the free parameters incorporated in $K$. Incidentally, any pair of orthonormal families $\phi, \psi$ on $(0, \infty)$ with $\phi=L \psi$, say, obviously induce a symmetric root $K$ through the matrix $L$ and any diagonal matrix $\operatorname{diag}\left((-1)^{r(m)}\right)$.

To return to our specific example, let $K_{1}, K_{2}$ be matrices of form (2.5) with characteristic parameters $c_{1}=q_{1}^{1 / 2}, c_{2}=q_{2}^{1 / 2}$ and a common value for $\alpha$. Then choosing

$$
a_{n}=(\Gamma(\alpha+n+1))^{\frac{1}{2}}(n ! \Gamma(\alpha+1))^{-\frac{1}{2}}, \quad n \geqq 0,
$$

we have at once from the identity

$$
\begin{aligned}
\sum_{n} \Gamma(1+\alpha+m+n)_{2} F_{1}\left(-m,-n ;-m-n-\alpha ; c^{-2}\right) z^{n} / n ! \\
=\Gamma(1+\alpha+m)\left(1-z c^{-2}\right)^{m}(1-z)^{-m-1-\alpha}
\end{aligned}
$$

that

$$
F_{p}^{K}(z)=s^{1+\alpha} c^{p}(\Gamma(\alpha+p+1))^{\frac{1}{2}}(p ! \Gamma(\alpha+1))^{-\frac{1}{2}}\left(1-z c^{-1}\right)^{p}(1-c z)^{-p-1-\alpha}
$$

and

$$
\begin{aligned}
F_{m}^{K_{1} K_{2}}(z)= & (-1)^{m}\left(s_{1} s_{2} /\left(1-c_{1} c_{2}\right)\right)^{1+\alpha}\left(\left(c_{2}-c_{1}\right) /\left(1-c_{1} c_{2}\right)\right)^{m} . \\
& (\Gamma(1+\alpha+m))^{\frac{1}{2}}(m ! \Gamma(1+\alpha))^{-\frac{1}{2}}\left[1-z\left(1-c_{1} c_{2}\right) /\left(c_{2}-c_{1}\right)\right]^{m} . \\
& {\left[1-z\left(c_{2}-c_{1}\right) /\left(1-c_{1} c_{2}\right)\right]^{-m-1-\alpha} . }
\end{aligned}
$$

Since

$$
\left(s_{1} s_{2}\right)^{2}+\left(c_{2}-c_{1}\right)^{2}=\left(1-c_{1} c_{2}\right)^{2},
$$

it follows that $K_{1} K_{2}$ is of the same form as $K$ (with parameter $c=$ $\left.\left(c_{2}-c_{1}\right) /\left(1-c_{1} c_{2}\right)\right)$ except for a minus sign attached to the entries in rows whose labels gave odd parity. Hence a product $K_{1} K_{2} K_{3}$ of three such matrices equals a fourth such matrix $K_{4}$ characterised by parameter

$$
c_{4}=\left(c_{1}-c_{2}+c_{3}-c_{1} c_{2} c_{3}\right) /\left(1-c_{1} c_{2}-c_{2} c_{3}+c_{3} c_{1}\right)
$$

From the symmetry between indices 1 and 3 we have incidentally that

$$
K_{1} K_{2} K_{3}=K_{3} K_{2} K_{1} .
$$

Setting $c_{1}=c_{3}, c_{4}=0$, we see that $K_{2}$ is diagonalised when $c_{2}=2 c_{1} /\left(1+c_{1}^{2}\right)$, that is, when $t_{1}=\left(2 t_{2}\right)^{\frac{1}{2}}$, in our earlier notation, as before. The relation

$$
K_{1} K_{2}=K_{4} K_{3}
$$

can again be expanded to yield bilinear relations derived by Meixner by complex variable methods. 
The union of the set of all $K$ and the set $H$ of all products of pairs of such elements constitutes a multiplicative group $G$. $H$ is a normal subgroup of $G$ and the corresponding factor group consists of two elements. The evaluations of third and higher order products of elements of $G$ provide further identities in the hypergeometric functions.

An exactly analogous argument holds for the matrices (3.4), starting from an easy demonstration that the product $K_{1} K_{2}$ of two matrices with parameters $c_{1}, c_{2}$ is another such matrix with parameter $c_{2}-c_{1}$ except in that entries in rows of odd parity carry a minus unity multiplier. The same result also follows from a limiting argument.

Since the similarity transform of a root of $I$ is also a root, we can furnish asymmetric roots from a matrix $S$ provided $S^{-1}$ can be exhibited explicitly. Guinand's discussion (1956), particularised to treat of square roots of $I$, exploits the fact that the matrix

$$
S=\left[\begin{array}{ccccc}
1 & 0 & 0 & 0 & . . \\
c_{1} & 1 & 0 & 0 & . . \\
c_{1} c_{2} / 2 ! & c_{2} & 1 & 0 & . . \\
c_{1} c_{2} c_{3} / 3 ! & c_{2} c_{3} / 2 ! & c_{3} & 1 & . . \\
. & . & . & . & . .
\end{array}\right]
$$

has an explicit inverse

$$
S^{-1}=\left[\begin{array}{ccccc}
1 & 0 & 0 & 0 & . \\
-c_{1} & 1 & 0 & 0 & \ldots \\
c_{1} c_{2} / 2 ! & -c_{2} & 1 & 0 & . \\
-c_{1} c_{2} c_{3} / 3 ! & c_{2} c_{3} / 2 ! & -c_{3} & 1 & \ldots \\
. & . & . & . & . .
\end{array}\right]
$$

to provide for the discovery of a very general class of square roots

$$
K=\left[\begin{array}{ccccc}
1 & 0 & 0 & 0 & . \\
2 c_{1} & -1 & 0 & 0 & . \\
4 c_{1} c_{2} / 2 ! & (-1) 2 c_{2} & (-1)^{2} & 0 & . \\
8 c_{1} c_{2} c_{3} / 3 ! & (-1) 4 c_{2} c_{3} / 2 ! & (-1)^{2} 2 c_{3} & (-1)^{3} & \\
. & . & . & . & .
\end{array}\right]
$$

from the basic root $\operatorname{diag}\left((-1)^{m}\right)$. 
In the same way the matrices $(2.5)$ are all transforms of diag $\left((-1)^{m}\right)$. Indeed, we have seen that any symmetric root can be obtained from an "elementary" diagonal root with the aid of an orthogonal matrix $X$. It is clear from the argument of section one that finding such a matrix $X$ is equivalent to determing a factorisation

$$
F^{I}(u, v)=\sum_{p=0}^{\infty} H_{p}^{X}(u) H_{p}^{X}(v),
$$

where $H$ is of the form

$$
H_{p}^{X}(u)=\sum_{m=0}^{\infty} x_{m p} a_{m} u^{m}
$$

with the helpful difference from that section that the matrix $X$ encapsulated in the generating functions $\left\{H_{p}^{X}(z), p \geqq 0\right\}$ is no longer required to be symmetric.

Straightforward solutions to this factorisation problem are obtained if one constructs orthogonal matrices from the five classical orthogonal polynomials in a discrete variable $y$ (see Erdélyi et al. (1953), volume 2). Of these, the Tchebychev and Krawtchouk systems are non-zero only for a finite range of values of $y$, and so give rise to roots $K$ which are the direct sum of an "elementary" root and a symmetric solution to the corresponding square root problem for finite matrices. For any of the remaining Meixner, Charlier and Hahn systems, let $\left\{\phi_{n}(y)\right\}$ represent the orthonormalised family. Then we have at once an associated system of symmetric roots given by

$$
k_{m n}=\sum_{y}(-1)^{r(y)} \phi_{m}(y) \phi_{n}(y), \quad r(y) \text { integral. }
$$

From our earlier discussion, these roots include ones already exhibited in the cases of the Meixner and Charlier families when $r(y)=y$. No such result holds for the Hahn family as the basic functions $\phi_{m}(x ; \beta, \gamma, \delta)$ do not possess symmetry between $m$ and $x$.

\section{References}

P. Barrucand (1950), 'Sur les suites réciproques', Comptes Rendus 230, 1727-1728.

S. Bochner (1929), 'Über Sturm-Liouvillesche Polynomsysteme', Math. Z. 29, 730-736.

R. G. Cooke (1950), Infinite Matrices and Sequence Spaces (Macmillan, London, 1950).

A. Erdélyi, W. Magnus, F. Oberhettinger and F. G. Tricomi (1953), Higher Transcendental Functions, Vols I-III (McGraw-Hill, New York, 1953).

A. Erdélyi, W. Magnus, F. Oberhettinger and F. G. Tricomi (1954), Tables of Integral Transforms, Vols I-II (McGraw-Hill, New York, 1954).

C. Fox (1929), 'A generalization of the Fourier-Bessel Integral transform', Proc. London Math. Soc. 29, 401-452. 
A. P. Guinand (1939), 'Finite summation formulae', Quart. J. Math. Oxford 10, 38-44.

A. P. Guinand (1942), 'Simple Fourier transforms', Quart. J. Math. Oxford 13, 153-158.

A. P. Guinand (1950), 'A class of Fourier kernels', Quart. J. Math. Oxford (2) 1, 191-193.

A. P. Guinand (1956), 'Matrices associated with fractional Hankel and Fourier transformations', Proc. Glasgow Math. Assoc. II, 185-192.

G. H. Hardy (1932), 'Summation of a series of polynomials of Laguerre', J. London Math. Soc. 7, 138-9 and 192.

G. H. Hardy and E. C. Titchmarsh (1933), 'A class of Fourier integrals', Proc. London Math. Soc. 35, $116-155$.

B. Kuttner (1934), 'Divisor functions: Fourier integral transforms', Proc. London Math. Soc. 37, 161-208.

C. C. MacDuffee (1946), The Theory of Matrices (Chelsea, New York, 1946).

J. Meixner (1941), 'Umformung gewisser Reihen, deren Glieder Produkte hypergeometrischer Funktionen sind', Deutsch Math. 6, 341-349.

S. Perlis (1952), Theory of Matrices (Addison-Wesley, Reading, Mass. 1952).

R. C. T. Smith (1972), 'An example of an orthogonal transformation of Hilbert sequence space', $J$. Austral. Math. Soc. 13, 357-361.

E. C. Titchmarsh (1937), Introduction to the Theory of Fourier Integrals (O.U.P. Oxford, 1937).

F. Tricomi (1948), Equazioni Differentiali (Torino, 1948).

G. N. Watson (1933a), 'General transforms', Proc. London. Math. Soc. 35, 156-199.

G. N. Watson (1933b), 'Notes on Generating Functions of polynomials: (1) Laguerre Polynomials', $J$. London Math. Soc. 8, 189-192.

Department of Applied Mathematics,

University of Adelaide,

Adelaide, 5001,

Australia. 